\title{
Peroxiredoxin-5 Knockdown Accelerates Pressure Overload- Induced Cardiac Hypertrophy in Mice
}

\author{
Chengyun Hu $\mathbb{D}^{1,2}$ Feibiao Dai ${ }^{1},{ }^{1,2}$ Jiawu Wang $\mathbb{D},{ }^{1}$ Lai Jiang $\mathbb{D},{ }^{3}$ Di Wang $\mathbb{D},{ }^{4}$ Jie Gao $\mathbb{D},{ }^{5}$ \\ Jun Huang $\mathbb{D}^{6}{ }^{6}$ Jianfeng Luo $\mathbb{D}^{7}$ Fei Tang $\mathbb{D}^{1},{ }^{1}$ Zhetao Zhang $\mathbb{D}^{8},{ }^{8}$ and Chaoliang Tang $\mathbb{D}^{1}$ \\ ${ }^{1}$ Department of Anesthesiology, The First Affiliated Hospital of USTC, Division of Life Sciences and Medicine, University of Science \\ and Technology of China, Hefei, Anhui 230001, China \\ ${ }^{2}$ Department of Anesthesia, Wannan Medical College, Wuhu, Anhui 241002, China \\ ${ }^{3}$ Department of Obstetrics and Gynecology, The First Affiliated Hospital of USTC, Division of Life Sciences and Medicine, \\ University of Science and Technology of China, Hefei, Anhui 230001, China \\ ${ }^{4}$ Department of Anesthesiology, The Second Affiliated Hospital of Anhui Medical University, Hefei, Anhui 230601, China \\ ${ }^{5}$ Department of Anesthesiology, The First Affiliated Hospital of Anhui Medical University, Hefei, Anhui 230022, China \\ ${ }^{6}$ Department of Anesthesiology, The People's Hospital of Chizhou, Chizhou, Anhui 247000, China \\ ${ }^{7}$ Department of Cardiology, The First Affiliated Hospital of USTC, Division of Life Sciences and Medicine, University of Science and \\ Technology of China, Hefei, Anhui 230001, China \\ ${ }^{8}$ Department of Pharmacy, The First Affiliated Hospital of USTC, Division of Life Sciences and Medicine, University of Science and \\ Technology of China, Hefei, Anhui 230036, China
}

Correspondence should be addressed to Fei Tang; ahsynqtf@126.com, Zhetao Zhang; ahsynqzzt@126.com, and Chaoliang Tang; chaolt@ustc.edu.cn

Received 19 August 2021; Accepted 21 December 2021; Published 29 January 2022

Academic Editor: Ji Bihl

Copyright ( $\odot 2022$ Chengyun Hu et al. This is an open access article distributed under the Creative Commons Attribution License, which permits unrestricted use, distribution, and reproduction in any medium, provided the original work is properly cited.

A recent study showed that peroxiredoxins (Prxs) play an important role in the development of pathological cardiac hypertrophy. However, the involvement of Prx 5 in cardiac hypertrophy remains unclear. Therefore, this study is aimed at investigating the role and mechanisms of Prx5 in pathological cardiac hypertrophy and dysfunction. Transverse aortic constriction (TAC) surgery was performed to establish a pressure overload-induced cardiac hypertrophy model. In this study, we found that Prx5 expression was upregulated in hypertrophic hearts and cardiomyocytes. In addition, Prx5 knockdown accelerated pressure overload-induced cardiac hypertrophy and dysfunction in mice by activating oxidative stress and cardiomyocyte apoptosis. Importantly, heart deterioration caused by Prx5 knockdown was related to mitogen-activated protein kinase (MAPK) pathway activation. These findings suggest that Prx5 could be a novel target for treating cardiac hypertrophy and heart failure.

\section{Introduction}

Pathological cardiac hypertrophy is a common pathophysiological process of various cardiovascular diseases, including hypertension, myocardial infarction, and heart failure [1-3]. It presents as thickening of the ventricular wall and decreased compliance of the ventricular wall [4-6]. Multiple mechanisms have been identified in the regulation of pathological cardiac hypertrophy, including oxidative stress, inflammation, autophagy, and cardiomyocyte apoptosis
[7-9]. Thus, targeting molecules or genes associated with the above processes is crucial for the treatment of pathological cardiac hypertrophy.

Peroxiredoxins (Prxs) are a superfamily of antioxidant peroxidases that scavenge hydrogen peroxide $\left(\mathrm{H}_{2} \mathrm{O}_{2}\right)$ and alkyl hydroperoxides [10-13]. At present, Prxs are identified as important regulators of redox homeostasis and participate in a series of cell functions. Many studies have shown that some family members of Prxs play an important role in the development of pathological cardiac hypertrophy [14, 
15]. Cardiomyocyte-specific Prx1 overexpression in mice attenuates cardiac hypertrophy and dysfunction under pressure overload [14]. Similarly, overexpression of Prx3 prevents cardiac hypertrophy and failure after myocardial infarction in mice [15]. As an important member of the Prx family, Prx5 has antioxidant protective functions and can effectively remove reactive oxygen species (ROS) [16, 17]. However, the involvement of Prx5 in cardiac hypertrophy remains unclear.

In this study, we explored the role and mechanisms of Prx5 in pathological cardiac hypertrophy and dysfunction. We confirmed that Prx5 knockdown accelerates pressure overload-induced cardiac hypertrophy and dysfunction in mice by activating oxidative stress and cardiomyocyte apoptosis. Importantly, heart deterioration caused by Prx 5 knockdown was related to mitogen-activated protein kinase (MAPK) activation. These findings suggest that Prx 5 could be a novel target for treating cardiac hypertrophy and heart failure.

\section{Materials and Methods}

2.1. Animals and Animal Model. All animal procedures in this study were approved by the Animal Care and Use Committee of Anhui Medical University. Male C57BL/6 mice (8-10 weeks, 23-26g) were purchased from Beijing HFK Bioscience Co., Ltd. (Beijing, China) and were housed in a well-ventilated environment. Transverse aortic constriction (TAC) surgery was performed to establish pressure overload-induced cardiac hypertrophy model as previously described [18]. Two weeks before TAC surgery, mice received a heart injection of AAV9-shPrx5 $\left(1 \times 10^{11}\right.$ viral particles/mouse) to knockdown Prx5 in the myocardium. Four weeks after TAC or the sham procedure, mice were euthanized by intraperitoneal injection of $200 \mathrm{mg} / \mathrm{kg}$ pentobarbital sodium. Then, the hearts, lungs, and tibia were harvested and measured to calculate the heart weight/body weight (HW/BW, mg/g), lung weight to body weight (LW/ $\mathrm{BW}, \mathrm{mg} / \mathrm{g}$ ), heart weight to tibia length (HW/TL, $\mathrm{mg} / \mathrm{mm}$ ), and lung weight/tibia length (LW/TL, $\mathrm{mg} / \mathrm{mm}$ ) ratios in the mice.

2.2. Echocardiography Analysis. Cardiac function was detected using a Vivid 7000 ultrasound equipped with a $14 \mathrm{MHz}$ transducer. In short, the mice were anesthetized, and the left ventricle (LV) geometry was assessed in both parasternal long-axis and short-axis views. The heart rate (HR), LV internal diameter at end-diastole (LVIDd), LV internal diameter at end-systole (LVIDs), LV posterior wall thickness of end systolic (LVPWs), LV posterior wall thickness of end diastolic (LVPWd), interventricular septal thickness at end-diastole (IVSd), interventricular septal thickness at end-systole (IVSs), and LV fractional shortening (LVFS) were determined.

2.3. Histological and TUNEL Analysis. The mice were sacrificed immediately after echocardiography measurements, and the hearts were harvested and then placed in $4 \%$ paraformaldehyde. Then, the heart sections were prepared and stained with hematoxylin and eosin (HE) and wheat germ agglutinin (WGA) for morphological analyses and evaluation of the cross-sectional area (CSA). In addition, heart sections were stained with picrosirius red (PSR) to assess collagen deposition. To detect cardiomyocyte apoptosis, TUNEL staining was performed as described in our previous study. The sections were visualized using microscopy, and all images were analyzed using Image-Pro Plus 6.0.

2.4. Neonatal Rat Cardiomyocyte (NRCM) Culture and Treatment. Primary neonatal rat cardiomyocytes (NRCMs) were isolated from 1- to 2-day-old Sprague-Dawley rats as previously described [19]. Then, the NRCMs were cultured in plating medium consisting of DMEM/F12 containing $15 \%$ fetal bovine serum (FBS), $0.1 \mathrm{mM} \mathrm{BrdU}$, and $100 \mathrm{mg} /$ $\mathrm{mL}$ penicillin/streptomycin. To knockdown Prx5 in vitro, Prx5 siRNA was used according to manufacturer's instructions. Then, the NRCMs were stimulated with angiotensin II (Ang II, $1 \mu \mathrm{M}$ ) for $48 \mathrm{~h}$.

2.5. Immunofluorescence Analysis. The NRCMs were fixed with $4 \%$ paraformaldehyde and permeabilized with $0.2 \%$ Triton X-100. Then, the NRCMs were stained with $\alpha$-actinin and the indicated fluorescent secondary antibody and then stained with DAPI. Finally, the NRCMs were visualized under a fluorescence microscope, and all images were analyzed using Image-pro Plus 6.0.

2.6. Quantitative Real-Time PCR. Total mRNA was extracted from ventricular tissue and NRCMs and then converted to cDNA using the RNA PCR Kit). PCR amplification was performed and quantified using an ABI PRISM 7000 Sequence Detection System. The relative mRNA expression levels of target genes were analyzed and normalized to the mRNA expression level of glyceraldehyde-3-phosphate dehydrogenase (GAPDH). The sequences of the primers used are presented in Table 1.

2.7. Western Blotting. Protein lysates of ventricular tissue and NRCMs were prepared and the protein concentrations were then measured. The proteins were loaded and run on SDS-PAGE and transferred to a PVDF membrane. The PVDF membranes were subsequently blocked with 5\% PVDF and incubated with primary antibodies against Prx5, GAPDH, Bax, Bcl-2, ERK, p-ERK, JNK, p-JNK, p38, and p-p38. After washing, the PVDF membranes were incubated with a secondary antibody and visualized with an infrared imaging system according to manufacturer's protocol. The specific protein expression levels were normalized to that of GAPDH.

2.8. Measurement of Oxidative Stress Level. Dihydroethidium (DHE) staining was performed according to manufacturer's protocol. In short, frozen sections of ventricular tissue were incubated with $10 \mu \mathrm{M}$ DHE in PBS in a humidified and light-protected chamber. The images were then taken with a laser microscope and analyzed using ImagePro Plus 6.0. In addition, superoxide dismutase (SOD), glutathione (GSH), malondialdehyde (MDA), and $\mathrm{H}_{2} \mathrm{O}_{2}$ in $\mathrm{LV}$ 
TABle 1: Primer sequences for RT-PCR assays.

\begin{tabular}{|c|c|c|c|}
\hline \multirow{3}{*}{$\begin{array}{l}\text { Gene } \\
\text { PRDX5 }\end{array}$} & \multirow{3}{*}{$\begin{array}{l}\text { Species } \\
\text { Mouse }\end{array}$} & \multicolumn{2}{|c|}{ Sequence $\left(5^{\prime}-3^{\prime}\right)$} \\
\hline & & Forward & GCTGCAAAGCCAGTTCTGTG \\
\hline & & Reverse & CCACTGAGGGAATGGCATCTC \\
\hline \multirow{2}{*}{ PRDX5 } & \multirow{2}{*}{ Rat } & Forward & GCAAGGTTCAGCTCCTGGCT \\
\hline & & Reverse & CAGGTGAGGCCTGTGCCATC \\
\hline \multirow{2}{*}{ ANP } & \multirow{2}{*}{ Mouse } & Forward & CCTGTGTACAGTGCGGTGTC \\
\hline & & Reverse & AAGCTGTTGCAGCCTAGTCC \\
\hline \multirow{2}{*}{ ANP } & \multirow{2}{*}{ Rat } & Forward & AAAGCAAACTGAGGGCTCTGCTCG \\
\hline & & Reverse & TTCGGTACCGGAAGCTGTTGCA \\
\hline \multirow{2}{*}{ BNP } & \multirow{2}{*}{ Mouse } & Forward & CTCAAGCTGCTTTGGGCACAAGAT \\
\hline & & Reverse & AGCCAGGAGGTCTTCCTACAACAA \\
\hline \multirow{2}{*}{$\mathrm{BNP}$} & \multirow{2}{*}{ Rat } & Forward & CAGCAGCTTCTGCATCGTGGAT \\
\hline & & Reverse & TTCCTTAATCTGTCGCCGCTGG \\
\hline \multirow{2}{*}{$\beta$-MHC } & \multirow{2}{*}{ Mouse } & Forward & TCTACCCAGCCAAGATCAAAGT \\
\hline & & Reverse & CCCATTCCTAATAAGCTGTGTGG \\
\hline \multirow{2}{*}{$\beta-\mathrm{MHC}$} & \multirow{2}{*}{ Rat } & Forward & TCTGGACAGCTCCCCATTCT \\
\hline & & Reverse & CAAGGCTAACCTGGAGAAGATG \\
\hline \multirow{2}{*}{ Myh7 } & \multirow{2}{*}{ Mouse } & Forward & ACTGTCAACACTAAGAGGGTCA \\
\hline & & Reverse & TTGGATGATTTGATCTTCCAGGG \\
\hline \multirow{2}{*}{ Myh7 } & \multirow{2}{*}{ Rat } & Forward & TGCTGTTATTGCTGCCATTG \\
\hline & & Reverse & AGGAGTTATCATTCCGAACTGTC \\
\hline \multirow{2}{*}{ TGF- $\beta$} & \multirow{2}{*}{ Mouse } & Forward & TGCTTCAGCTCCACAGAGAA \\
\hline & & Reverse & TGGTTGTAGAGGGCAAGGAC \\
\hline TGF- $\beta$ & Rat & Forward & ATTCCTGGCGTTACCTTGG \\
\hline 1 UT $-p$ & Kal & Reverse & AGCCCTGTATTCCGTCTCCT \\
\hline CTGF & Moure & Forward & TGTGTGATGAGCCCAAGGAC \\
\hline CIGF & Mrouse & Reverse & AGTTGGCTCGCATCATAGTTG \\
\hline CTGF & Bat & Forward & ACACAAGGGTCTCTTCTGCG \\
\hline CIGF & Kat & Reverse & ACAGGGTGCACCATCTTTGG \\
\hline Colloren I & Monse & Forward & TGGTACATCAGCCCGAAC \\
\hline Conagen 1 & Mrouse & Reverse & GTCAGCTGGATAGCGACA \\
\hline Colloren I & Pot & Forward & TGGACATTAGGCGCAGGAA \\
\hline Conagen 1 & Kal & Reverse & GAGAGAGCATGACCGATGGATT \\
\hline Colloron UI & Mons & Forward & ACGTAGATGAATTGGGATGCAG \\
\hline Conagen 111 & Mrouse & Reverse & GGGTTGGGGCAGTCTAGTC \\
\hline$C$ llon & Dot & Forward & CCCAACCCAGAGATCCCATT \\
\hline Collagen 111 & Kat & Reverse & GAAGCACAGGAGCAGGTGTAGA \\
\hline 18 & Monco & Forward & GGGCCTCAAAGGAAAGAATC \\
\hline $1 L-1 \beta$ & Mouse & Reverse & TACCAGTTGGGGAACTCTGC \\
\hline U 6 & & Forward & AGTTGCCTTCTTGGGACTGA \\
\hline IL-6 & Mouse & Reverse & TCCACGATTTCCCAGAGAAC \\
\hline U 17 & Monec & Forward & TCCAGAAGGCCCTCAGACTA \\
\hline $1 \mathrm{~L}-\mathrm{I} /$ & Mouse & Reverse & AGCATCTTCTCGACCCTGAA \\
\hline $\mathrm{TNF}_{-} \alpha$ & Mouse & Forward & CCCAGGGACCTCTCTCTAATC \\
\hline $1 N F-\alpha$ & Mouse & Reverse & ATGGGCTACAGGCTTGTCACT \\
\hline GAPDH & Mouse & Forward & AACTTTGGCATTGTGGAAGG \\
\hline UAFDI & Noure & Reverse & CACATTGGGGGTAGGAACAC \\
\hline & & Forward & GACATGCCGCCTGGAGAAAC \\
\hline GAPDH & Rat & Reverse & GACATGCCGCCTGGAGAAAC \\
\hline
\end{tabular}



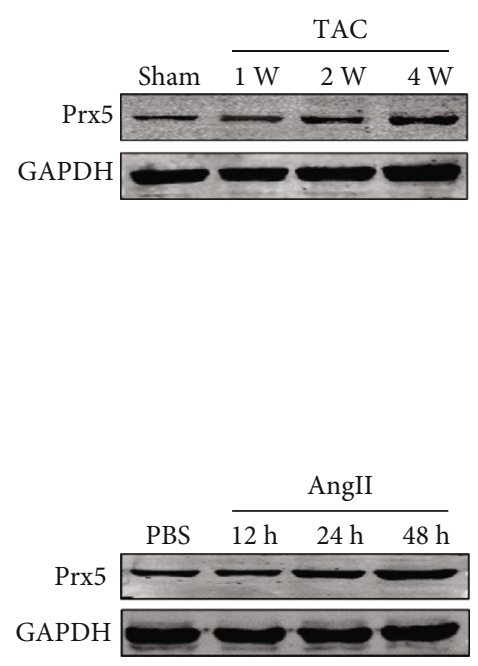

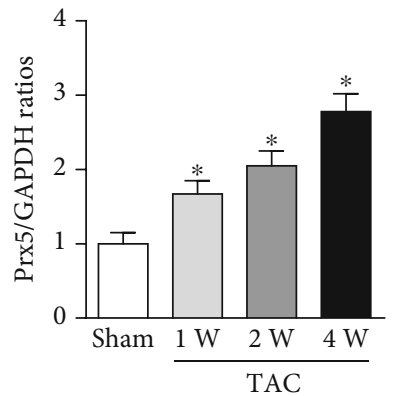

(a)

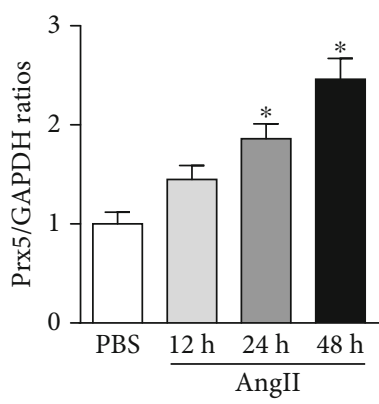

(b)
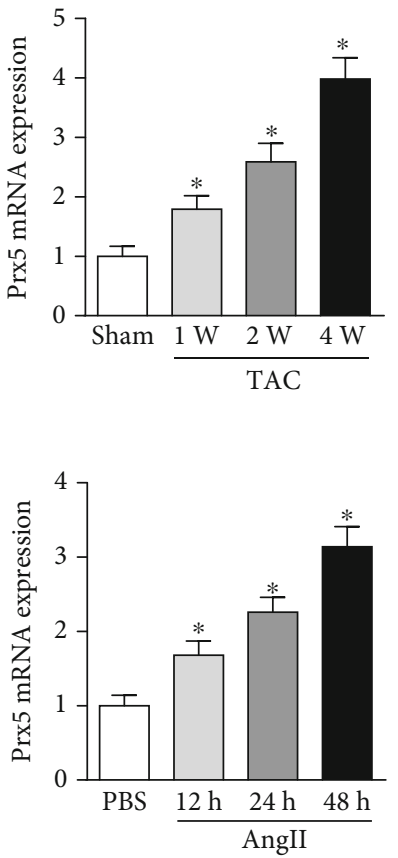

Figure 1: Prx5 expression is increased in hypertrophic hearts and isolated NRCMs. (a) The expression of Prx5 in heart tissues was measured by Western blotting and RT-PCR ( $n=4,{ }^{*} P<0.05$ vs. the sham group). (c) The expression of Prx 5 in heart tissues was measured by Western blotting and RT-PCR ( $n=4,{ }^{*} P<0.05$ vs. the PBS group).

TABLE 2: Echocardiographic data of each group.

\begin{tabular}{lcccc}
\hline Groups & Sham+AAV9-shRNA & Sham+AAV9-shPrx5 & TAC+ AAV9-shRNA & TAC+AAV9-shPrx5 \\
\hline HR (bpm) & $523 \pm 48$ & $519 \pm 39$ & $511 \pm 40$ & $516 \pm 44$ \\
LVEDd (mm) & $3.51 \pm 0.23$ & $3.63 \pm 0.35$ & $4.51 \pm 0.36 *$ & $5.32 \pm 0.29^{\#}$ \\
LVEDs (mm) & $1.93 \pm 0.16$ & $1.88 \pm 0.12$ & $3.18 \pm 0.21 *$ & $4.23 \pm 0.29^{\#}$ \\
LVPWd (mm) & $0.66 \pm 0.06$ & $0.64 \pm 0.05$ & $1.21 \pm 0.11 *$ & $1.43 \pm 0.13^{\#}$ \\
LVPWs (mm) & $1.06 \pm 0.10$ & $1.05 \pm 0.09$ & $1.17 \pm 0.09 *$ & $2.33 \pm 0.15^{\#}$ \\
IVSd (mm) & $0.64 \pm 0.05$ & $0.63 \pm 0.06$ & $1.82 \pm 0.15 *$ & $1.46 \pm 0.13^{\#}$ \\
IVSs (mm) & $1.06 \pm 0.11$ & $1.04 \pm 0.10$ & $29.76 \pm 3.03 *$ & $2.24 \pm 0.16^{\#}$ \\
FS (\%) & $45.41 \pm 2.68$ & $46.53 \pm 2.81$ & $20.34 \pm 2.54^{\#}$ \\
\hline
\end{tabular}

$n=8$ for each group. ${ }^{*} P<0.05$ vs. the sham group; ${ }^{\#} P<0.05$ vs. the TAC group.

tissue or NRCMs were detected using kits purchased from Beyotime Biotechnology Corporation (China).

2.9. Statistical Analysis. The data are expressed as the mean \pm standard deviation. Comparisons between two groups were analyzed using an unpaired Student's $t$ test. Differences among multiple groups were assessed using an analysis of variance followed by one-way analysis of variance. A value of $P<0.05$ was considered statistically significant.

\section{Results}

3.1. Prx5 Expression Is Increased in Hypertrophic Hearts and Isolated NRCMs. The results showed that Prx5 expression was gradually upregulated in the hearts of mice subjected to TAC surgery (Figure 1(a)). In accordance with this, higher Prx5 levels were also detected in isolated NRCMs after Ang II stimulation (Figure 1(b)). Together, these data suggest that Prx5 may participate in the development of cardiac hypertrophy.

3.2. Prx5 Knockdown Accelerates Pressure Overload-Induced Cardiac Dysfunction. After TAC surgery, animals exhibited LV dilatation and thickening, as indicated by increased LVIDd, LVIDs, LVPWd, LVPWs, IVSd, and IVSs and decreased FS. However, Prx 5 knockdown further aggravated pressure overload-induced cardiac dysfunction (Table 2). In addition, there were no significant differences in HR among the four groups.

3.3. Prx5 Knockdown Accelerates Pressure Overload-Induced Cardiac Hypertrophy. As shown in Figure 2, AAV9-shPrx5 caused decreased expression of Prx5 in hearts (Figure 2(a)). Four weeks after TAC surgery, Prx 5 knockdown accelerated 

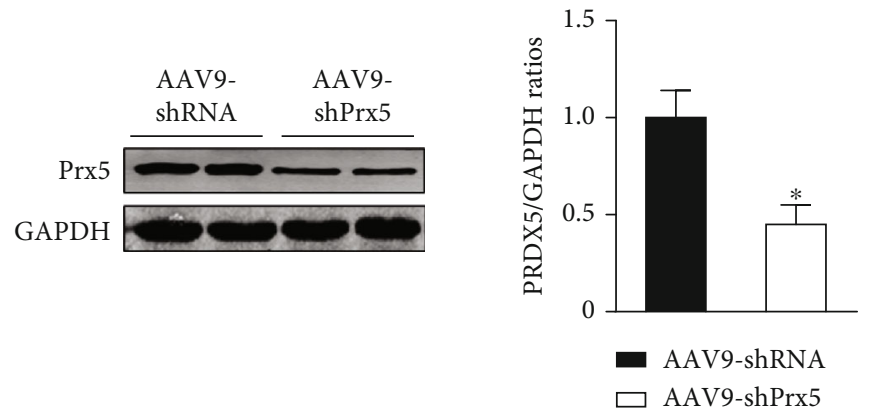

(a)
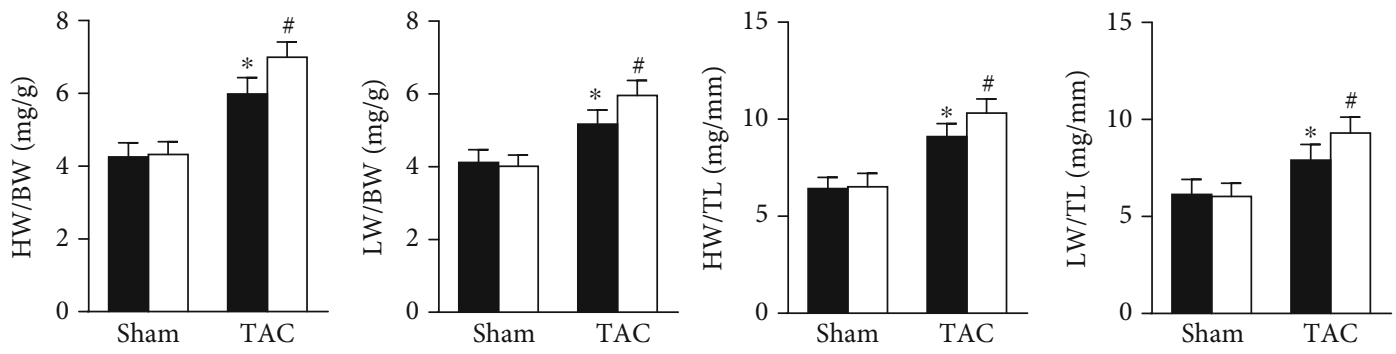

(b)
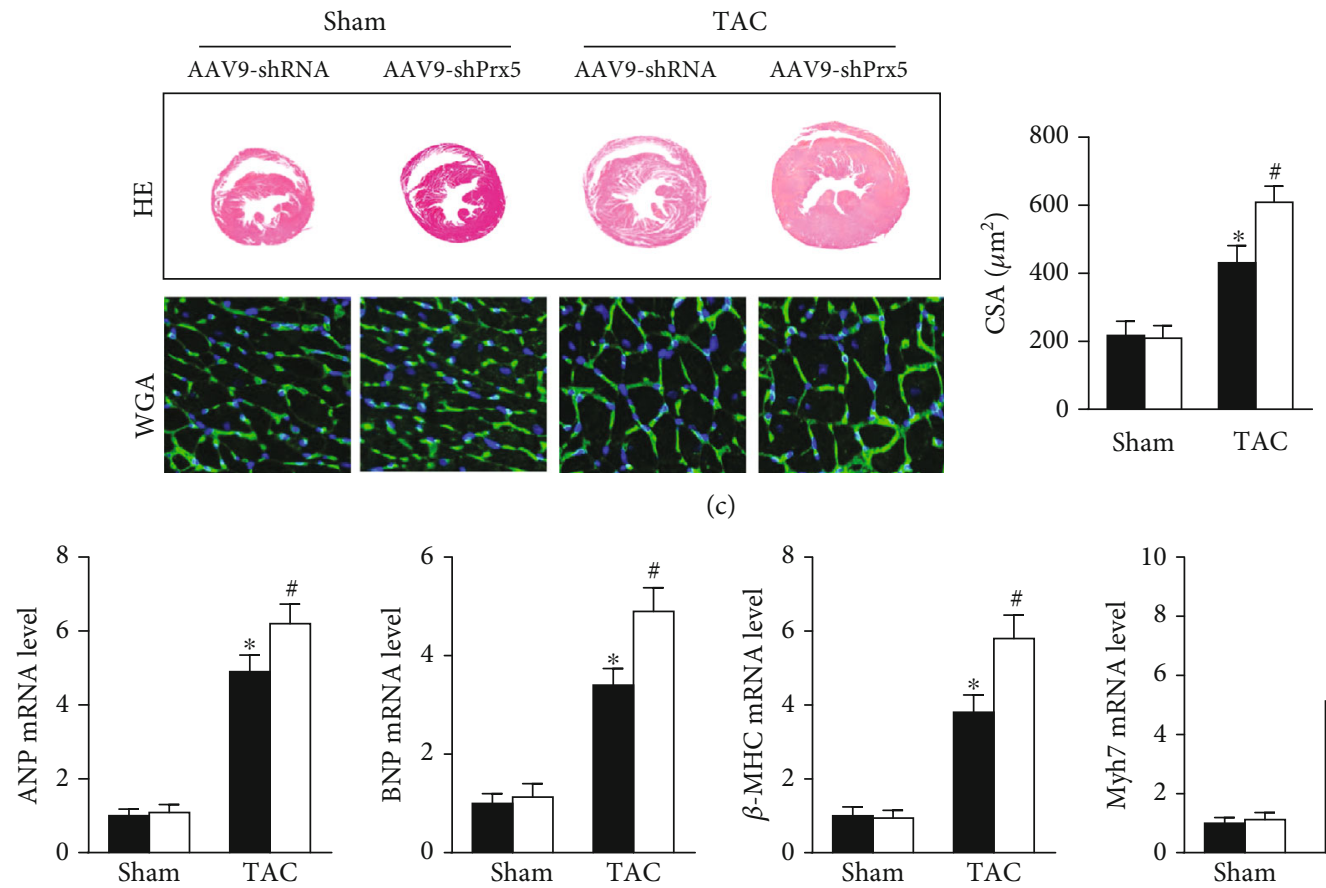

(c)
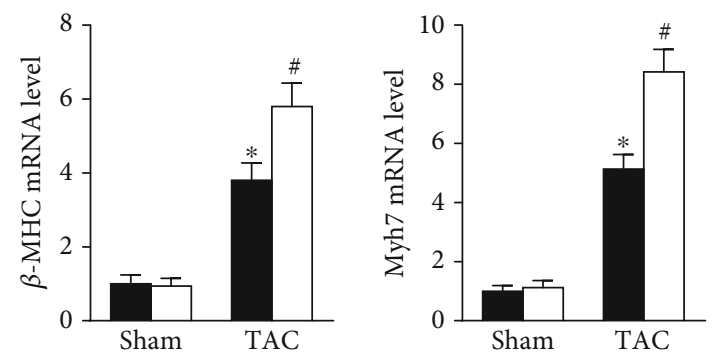

(d)

Figure 2: Prx5 knockdown accelerates pressure overload-induced cardiac hypertrophy. (a) The levels of Prx5 after injection with AAV9shPrx5 $(n=4)$. (b) Results for the HW/BW ratio, HW/TL ratio, LW/BW ratio, LW/TL ratio, and CSA of each group ( $n=6)$. (c) HE and WGA staining were performed in each group $(n=6$; scale bar, $50 \mu \mathrm{m})$. (d) The expression of ANP, BNP, $\beta$-MHC, and Myh7 was measured by RT-PCR in each group $(n=5)$. ${ }^{*} P<0.05$ vs. the sham group; ${ }^{\sharp} P<0.05$ vs. the TAC group.

pressure overload-induced cardiac hypertrophy, as evidenced by increased HW/BW, HW/TL, LW/BW, and LW/TL ratios and increased CSA (Figures 2(b) and 2(c)). In addition, higher mRNA levels of atrial natriuretic peptide (ANP), brain natriuretic peptide (BNP), $\beta$-myosin heavy chain $(\beta$-MHC), and Myosin Heavy Chain 7 (Myh7) were also found in the Prx5 knockdown group after TAC surgery (Figure 2(c)).
3.4. Prx5 Knockdown Accelerates Pressure OverloadInduced Cardiac Fibrosis. As shown in Figure 3, dramatic collagen deposition was observed in the mice after TAC surgery and was further increased in the Prx 5 knockdown group (Figure 3(a)). Similarly, after TAC surgery, the mRNA expression levels of several fibrosis markers, including collagen I, collagen III, transforming growth 

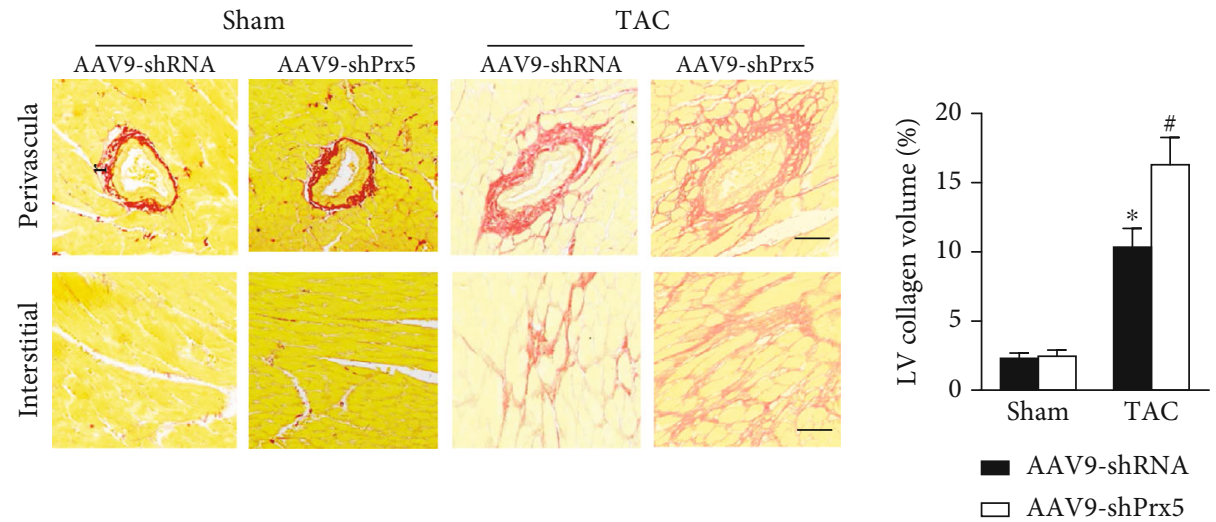

(a)
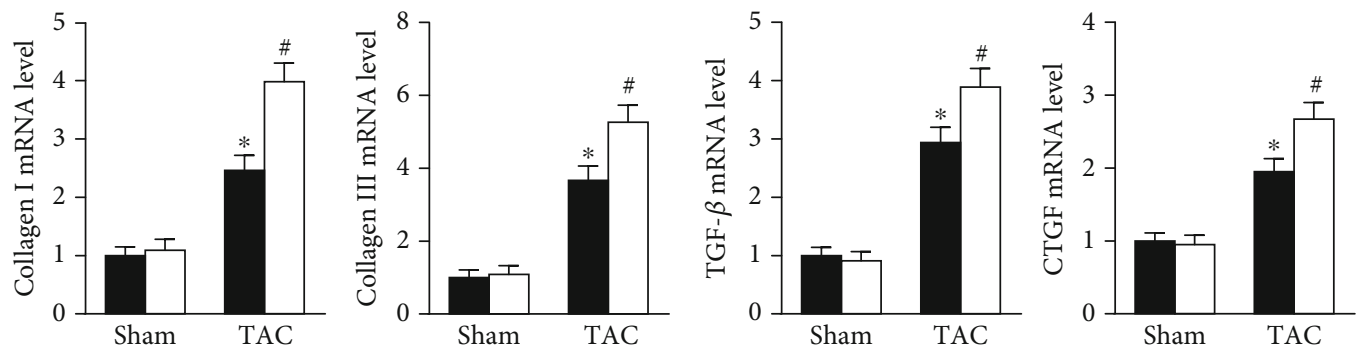

(b)

FIgURE 3: Prx5 knockdown accelerates pressure overload-induced cardiac fibrosis. (a) PSR staining was performed in each group ( $n=6$; scale bar, $50 \mu \mathrm{m}$ ). (b) The expression of collagen I, collagen III, TGF- $\beta$, and CTGF was measured by RT-PCR in each group ( $n=6)$. ${ }^{*} P<0.05$ vs. the sham group; ${ }^{\#} P<0.05$ vs. the TAC group.
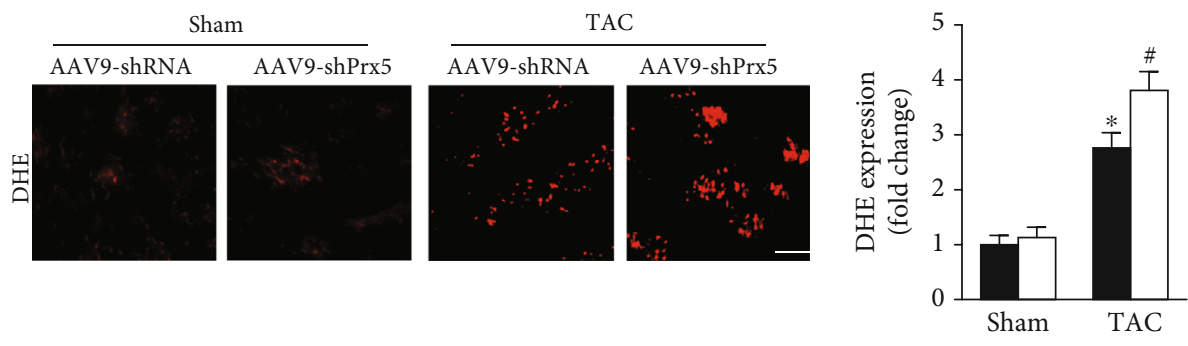

- AAV9-shRNA $\square$ AAV9-shPrx5

(a)
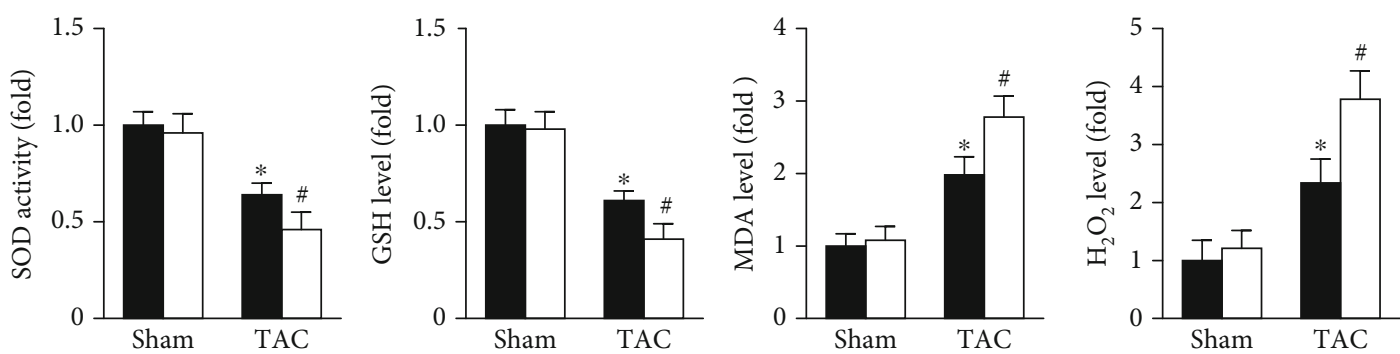

(b)

Figure 4: Prx5 knockdown accelerates pressure overload-induced oxidative stress and apoptosis. (a) DHE staining was performed in each group $(n=5$; scale bar, $100 \mu \mathrm{m})$. (b) Quantitative results of SOD activity and GSH, MDA, and $\mathrm{H}_{2} \mathrm{O}_{2}$ levels in the hearts of each group ( $n=6$ ). ${ }^{*} P<0.05$ vs. the sham group; ${ }^{\#} P<0.05$ vs. the TAC group. 

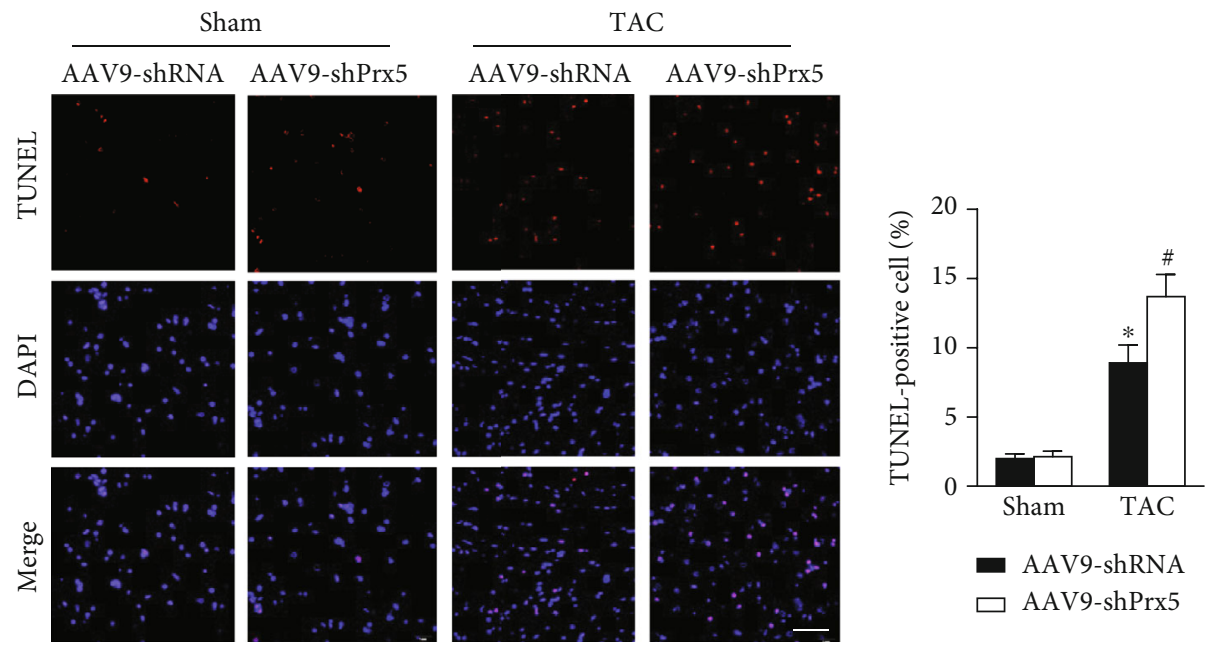

(a)
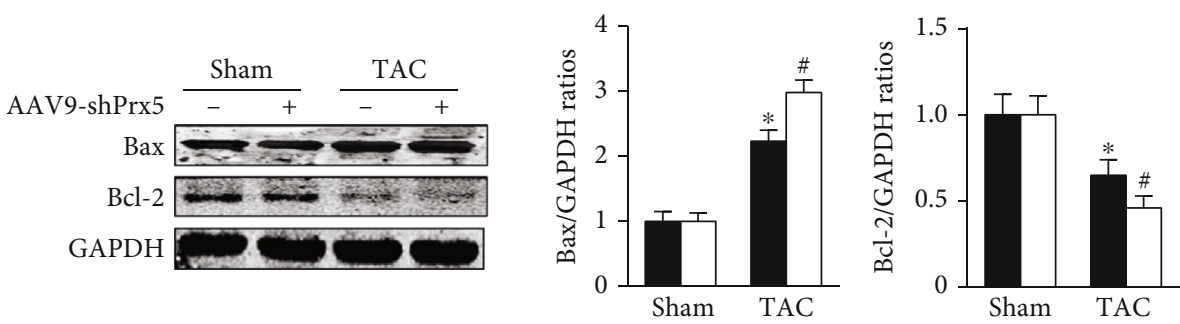

(b)

Figure 5: Prx5 knockdown accelerates pressure overload-induced apoptosis in mice. (a) TUNEL staining was performed in each group $(n=4$; scale bar, $50 \mu \mathrm{m})$. (b) The expression of Bax and Bcl-2 was measured by Western blot in each group $(n=4)$. ${ }^{*} P<0.05$ vs. the sham group; ${ }^{\#} P<0.05$ vs. the TAC group.

factor (TGF)- $\beta$, and connective tissue growth factor (CTGF), were also further increased in the Prx5 knockdown group (Figure 3(b)).

3.5. Prx5 Knockdown Accelerates Pressure OverloadInduced Oxidative Stress and Apoptosis in Mice. DHE staining was used to evaluate in vivo oxidative stress levels. The results showed that the oxidative stress level was dramatically increased in the Prx5 knockdown group after TAC surgery (Figure 4(a)). Moreover, Prx5 knockdown markedly decreased SOD activity and GSH levels and increased MDA and $\mathrm{H}_{2} \mathrm{O}_{2}$ levels in TAC-treated mice (Figure 4(b)).

3.6. Prx5 Knockdown Accelerates Pressure Overload-Induced Apoptosis in Mice. TUNEL staining was used to evaluate apoptosis levels in the heart. The results showed that the number of TUNEL-positive cells was dramatically increased in the Prx5 knockdown group after TAC surgery (Figure 5(a)). Moreover, Prx5 knockdown increased Bax and decreased Bcl-2 levels in TAC-treated mice (Figure 5(b)).

\subsection{Prx5 Knockdown Accelerates AngII-Induced} Cardiomyocyte Hypertrophy In Vitro. Consistent with the in vivo results, si-Prx5 led to decreased expression of Prx5 in NRCMs (Figure 6(a)). After $48 \mathrm{~h}$ of AngII stimulation, the NRCMs exhibited clear hypertrophy by augmentation in CSA and increased mRNA levels of ANP, BNP, $\beta$ MHC, and Myh7 (Figures 6(b) and 6(c)). Interestingly, Prx 5 knockdown accelerated AngII-induced cardiomyocyte hypertrophy in vitro (Figures 6(b) and 6(c)).

\subsection{Prx5 Knockdown Accelerates AngII-Induced Oxidative} Stress and Apoptosis In Vitro. The results showed that AngII treatment markedly decreased SOD activity and GSH levels and increased $\mathrm{MDA}$ and $\mathrm{H}_{2} \mathrm{O}_{2}$ levels in vitro, while these effects were further augmented by Prx 5 knockdown (Figure $7(\mathrm{a})$ ). TUNEL staining further showed that Prx5 knockdown further increased the number of TUNELpositive cells in vitro (Figure 7(b)).

3.9. Effect of Prx5 on the MAPK Signaling Pathway. Previous research has widely implicated MAPK signaling in cardiac hypertrophy. Thus, we investigated whether the effects of Prx5 are associated with the MAPK signaling pathway. The results showed that the phosphorylated levels of ERK, p38, and JNK were significantly increased in TAC-treated mice. However, these effects were further augmented by Prx 5 knockdown (Figure 8(a)). Consistent with the in vivo results, Prx5 knockdown also increased the phosphorylation levels of ERK, p38, and JNK in NRCMs after AngII treatment (Figure 8(b)). 

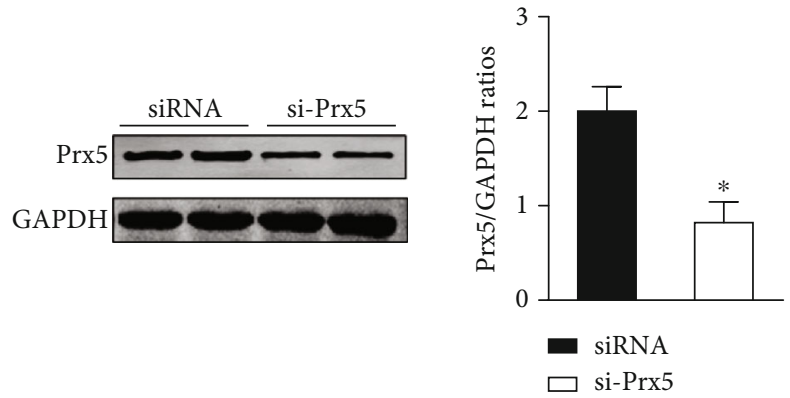

(a)
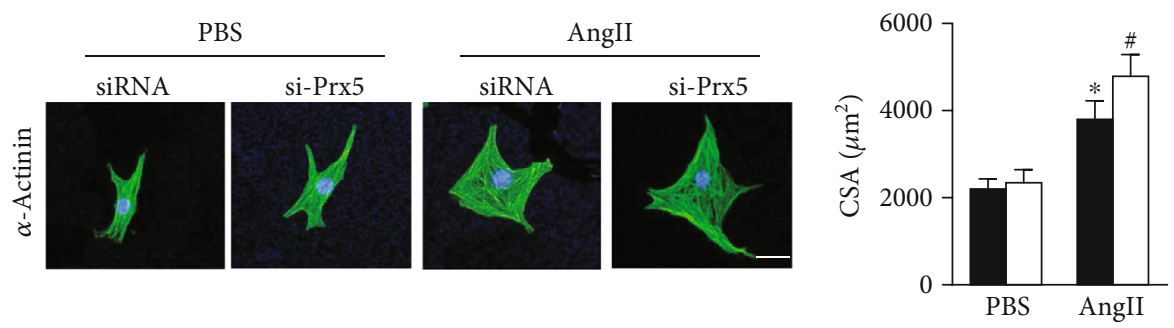

(b)
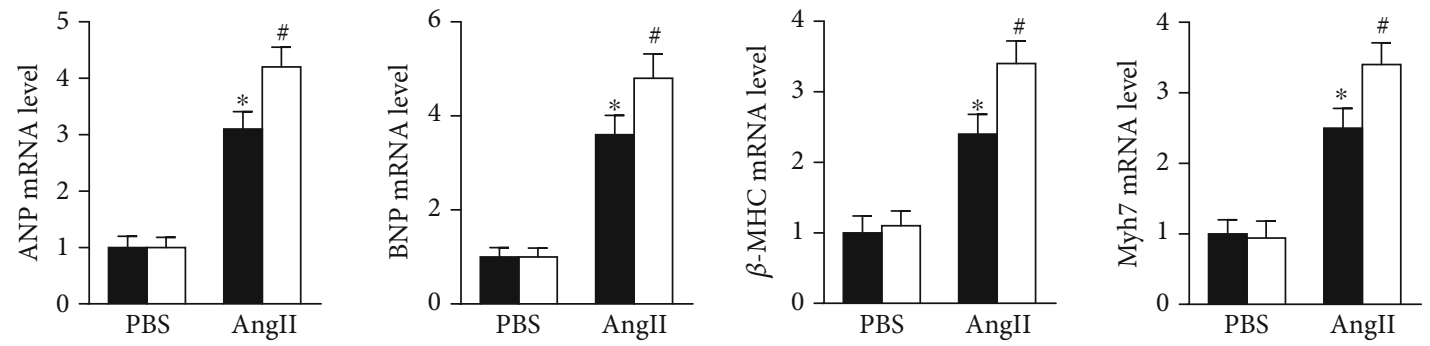

(c)

Figure 6: Prx5 knockdown accelerates AngII-induced cardiomyocyte hypertrophy in vitro. (a) The levels of Prx5 were measured by Western blot $(n=4)$. (b) Immunofluorescence staining for $\alpha$-actinin was performed in each group $(n=4$; scale bar, $25 \mu \mathrm{m})$. (c) The expression of ANP, BNP, $\beta$-MHC, and Myh7 was measured by RT-PCR in each group $(n=6) .{ }^{*} P<0.05$ vs. the PBS group; ${ }^{*} P<0.05$ vs. the AngII group.

\section{Discussion}

In the present study, we uncovered a novel role of Prx5 in pressure overload-induced cardiac hypertrophy. The results showed that the mRNA and protein expression of Prx5 were noticeably upregulated in hypertrophic hearts and AngIIstimulated cardiomyocytes. In addition, Prx5 knockdown accelerated pressure overload-induced cardiac hypertrophy and dysfunction in mice by activating oxidative stress and cardiomyocyte apoptosis. Importantly, heart deterioration caused by Prx 5 knockdown was related to MAPK activation. These findings suggest that Prx5 could be a novel target for treating cardiac hypertrophy and heart failure.

Prx5, also called PrxV/AOEB166/PMP20/PLP/ACR1, was discovered twenty years ago and is widely expressed in mammalian tissues [20]. As an important member of the Prxs family, Prx5 plays a central role in redox signal transduction and exhibits high scavenging activity toward oxidative stress [16] [21]. Previous research has shown that Prx5 exhibits a protective role in a variety of diseases, including brain lesions, aging, obesity, and cancer [22-24]. Recombinant Prx5 administration provided protection against $\mathrm{N}$ methyl-D-aspartate-mediated brain lesions and neuronal death in newborn mice [22]. In an obesity model induced by a high-fat diet, deletion of Prx 5 increased susceptibility to obesity and adipogenesis by increasing ROS generation and adipogenic gene expression [23]. However, little is known about the exact role and mechanisms of Prx 5 in the development of cardiac hypertrophy and dysfunction. In the present study, we first confirmed that Prx5 was upregulated in hypertrophic mouse hearts and AngII-stimulated NRCMs, indicating that Prx5 might be involved in the progression and development of pathological cardiac hypertrophy. In addition, AAV9-shPrx5 was used to knock down Prx5 in the myocardium. The results showed that Prx5 knockdown accelerates pressure overload-induced cardiac dysfunction, hypertrophy, and fibrosis in mice. Consistent with the in vivo results, Prx5 knockdown also accelerated AngII-induced cardiomyocyte hypertrophy in vitro.

Oxidative stress is described as a common pathological feature of various cardiovascular diseases [25-28]. As a natural byproduct of the metabolic utilization of oxygen, ROS are oxygen-containing molecules with highly reactive properties and represent crucial drivers of oxidative stress [29-31]. Under pathological conditions of pressure overload, excessive ROS result in cardiomyocyte death or 

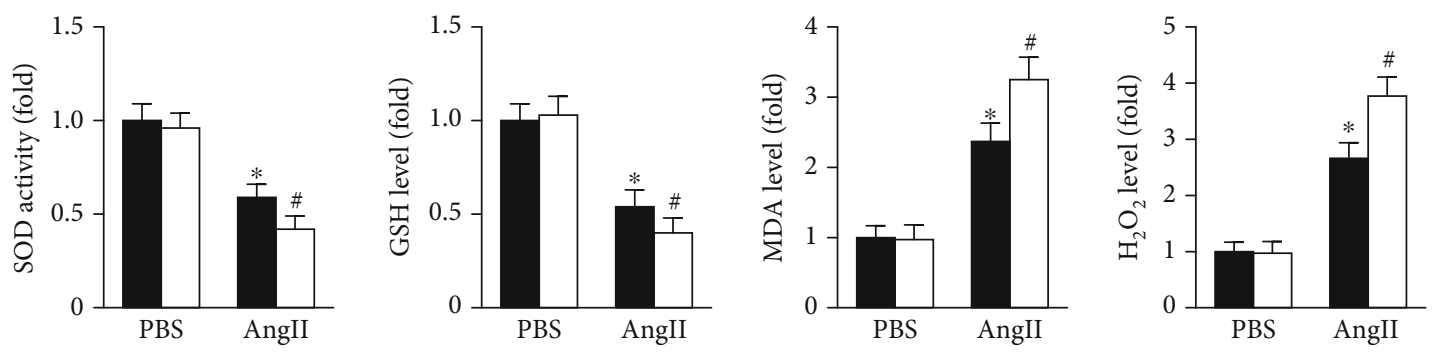

(a)
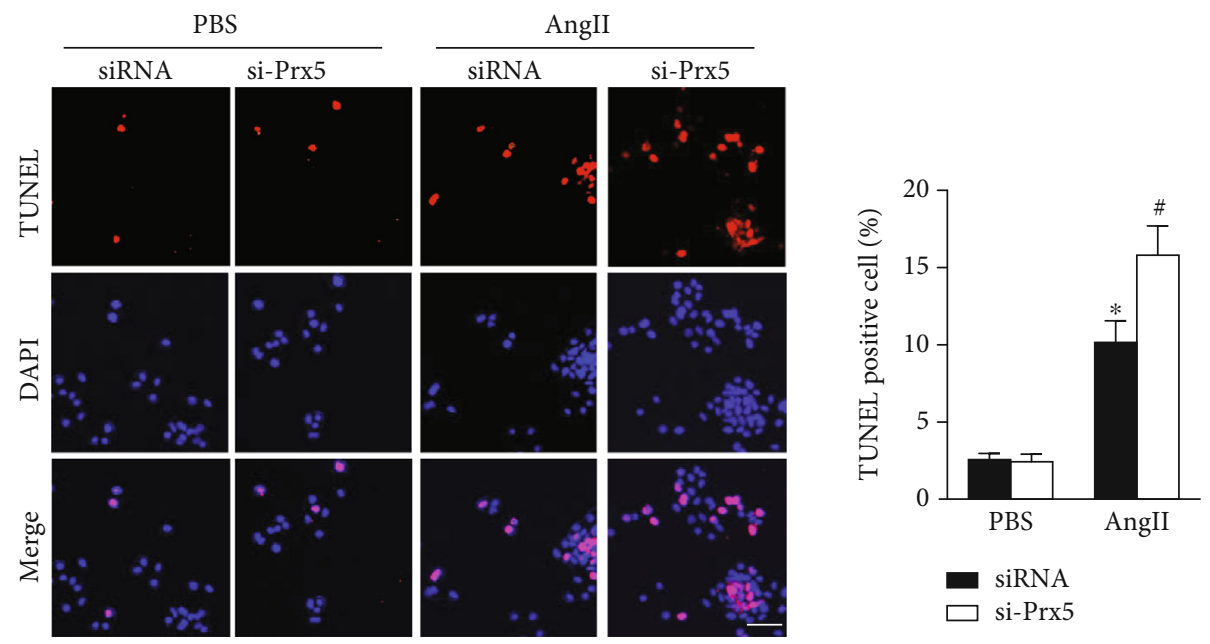

(b)

FIgure 7: Prx5 knockdown accelerates AngII-induced oxidative stress and apoptosis in vitro. (a) Quantitative results of SOD activity and $\mathrm{GSH}, \mathrm{MDA}$, and $\mathrm{H}_{2} \mathrm{O}_{2}$ levels in the hearts of each group $(n=6)$. (c) TUNEL staining was performed in each group ( $n=4$; scale bar, $50 \mu \mathrm{m}) .{ }^{*} P<0.05$ vs. the PBS group; ${ }^{\#} P<0.05$ vs. the AngII group.

functional damage and ultimately cardiac dysfunction [32-34]. There is likely benefit from the suppression of oxidative stress and countering excessive production of ROS in pathological cardiac hypertrophy therapy.

Many studies have shown that Prx5 has antioxidant protective functions and can effectively remove oxidative stress [17] [23]. Thus, we investigated whether Prx5 is involved in the occurrence of cardiac hypertrophy and dysfunction by regulating oxidative stress. The results showed that Prx 5 knockdown markedly decreased SOD activity and GSH levels and increased MDA and $\mathrm{H}_{2} \mathrm{O}_{2}$ levels in TAC-treated mice. DHE staining results also showed that the oxidative stress level was dramatically increased in the Prx5 knockdown group after TAC surgery. Consistent with the in vivo results, Prx 5 knockdown accelerated AngII-induced oxidative stress in vitro. These results indicate that the deterioration effect of Prx 5 knockdown is related to oxidative stress.

Apoptosis is known to contribute to various cardiovascular diseases, including heart failure, myocardial infarct, and reperfusion injury $[35,36]$. Previous research has also shown that cardiac hypertrophy is related to a reduced cell number due to enhanced apoptosis $[37,38]$. In addition, oxidative stress has been shown to be responsible for cardiomyocyte apoptosis [39-41]. Thus, we asked whether Prx5 affects cardiomyocyte apoptosis in pathological cardiac hypertrophy. The results showed that the expression of Bax was upregulated, the expression of $\mathrm{Bcl}-2$ was lower, and there were more TUNEL-positive cells in the TAC group than in the sham group. These effects were further augmented by Prx 5 knockdown. In addition, the results further confirmed the in vitro cell experiments, indicating that the deterioration effect of Prx5 knockdown is associated with cardiomyocyte apoptosis.

As intracellular signaling proteins, MAPKs have been shown to regulate various cellular processes, including cell size, cell growth, and cell survival, in response to extracellular stimuli [41-43]. It is well established that the activation of MAPK signaling increases cardiac damage and exacerbates pathological cardiac hypertrophy [44-46]. In this study, Prx5 knockdown accelerated pressure overloadinduced cardiac hypertrophy and dysfunction. However, the role of Prx5 in MAPK signaling activation in pathological cardiac hypertrophy was unclear. Thus, we examined the phosphorylation and total expression of ERK/JNK/p38 in hypertrophic hearts and AngII-stimulated cardiomyocytes. The results showed that Prx 5 knockdown significantly induced the phosphorylation of ERK/JNK/p38 in the TAC group, but the expression of total ERK/JNK/p38 remained unchanged. These results indicate that the deterioration effect of Prx 5 knockdown is associated with activation of MAPK signaling.

4.1. Clinical Significance. Pathological cardiac hypertrophy is a common pathophysiological process of various 

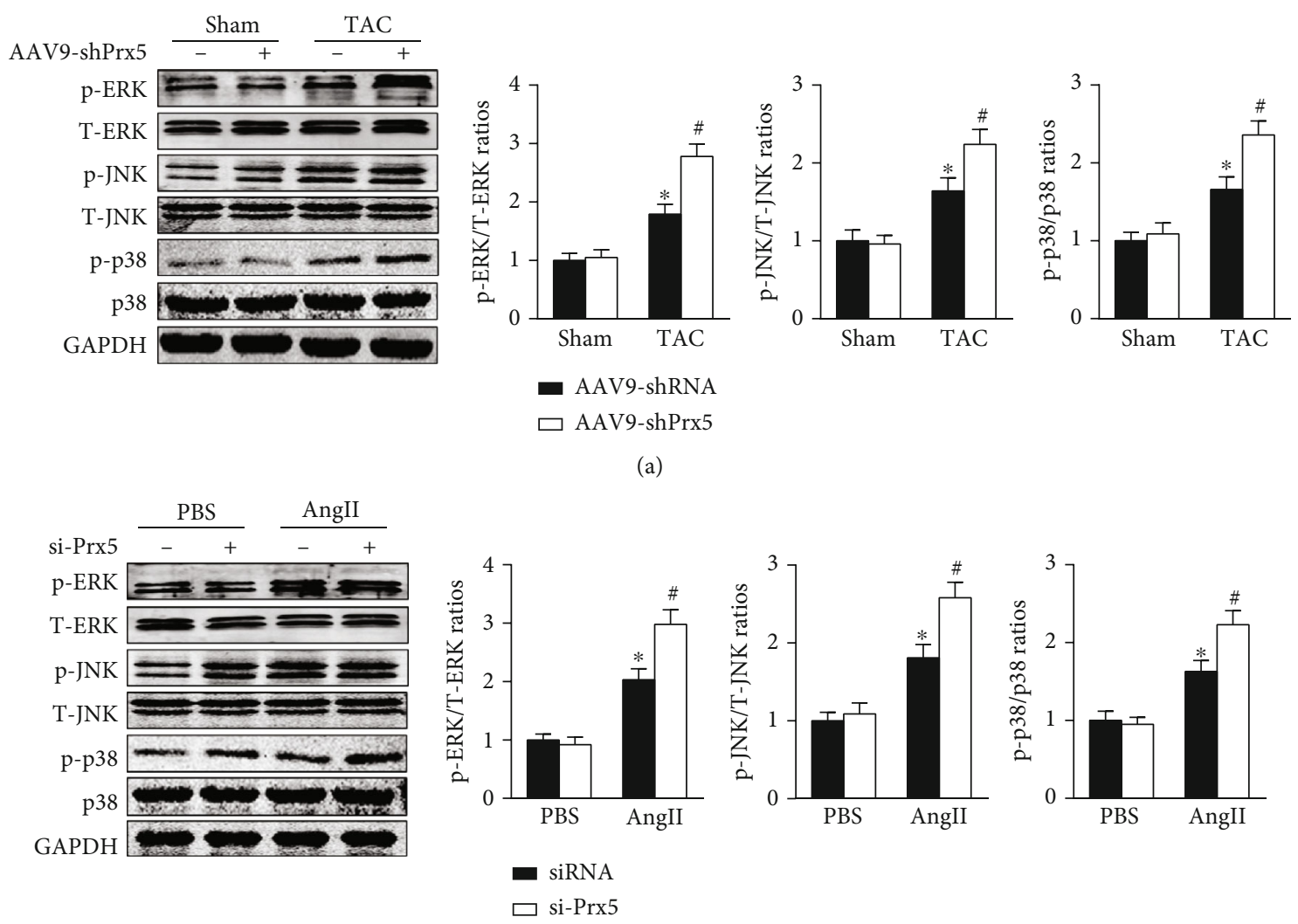

(b)

Figure 8: Effect of Prx5 on the MAPK signaling pathway. (a) The expression of p-ERK, t-ERK, p-JNK, t-JNK, p-p38, and p38 in the heart was measured by Western blotting $(n=4)$. (b) The expression of p-ERK, ERK, p-JNK, JNK, p-p38, and p38 in NVCMs was measured by Western blotting $(n=4) .{ }^{*} P<0.05$ vs. the sham or PBS group; ${ }^{\#} P<0.05$ vs. the TAC or AngII group.

cardiovascular diseases, including hypertension, myocardial infarction, and heart failure. Currently, there is no specific treatment to effectively reverse cardiac pathological hypertrophy and reduce the morbidity and mortality of heart failure. In this study, we demonstrated that Prx5 knockdown accelerated pressure overload-induced cardiac hypertrophy and dysfunction in mice by activating oxidative stress and cardiomyocyte apoptosis. Importantly, heart deterioration caused by Prx 5 knockdown was related to MAPK activation. These findings provided a new target for the prevention and treatment of cardiac hypertrophy and heart failure.

4.2. Study Limitations. This study was subject to the following limitations. First, as pathological cardiac hypertrophy is a multifactorial syndrome, we cannot exclude the possibility that Prx5 utilizes other pathways to protect the heart under pressure overload. Thus, more research is needed to determine the mechanism underlying the cardioprotective effects of Prx5. In addition, in our study, mice received a heart injection of AAV9-shPrx 5 to knock down Prx5 in the myocardium. However, animals with cardiac-specific overexpression or knockout of Prx5 may more precisely demonstrate the important function of Prx5 in pathological cardiac hypertrophy and dysfunction.

Taken together, our results have uncovered novel insights into the regulation of pathological cardiac hypertro- phy and dysfunction by Prx5. The results showed that Prx5 knockdown accelerates pressure overload-induced cardiac hypertrophy and dysfunction. Our data indicate that Prx5 may be an attractive target for the prevention and treatment of pathological cardiac hypertrophy and heart failure.

\section{Data Availability}

We declare that the materials described in the manuscript, including all relevant raw data, will be freely available to any scientist wishing to use them for noncommercial purposes, without breaching participant confidentiality.

\section{Conflicts of Interest}

No conflicts of interest are declared by the authors.

\section{Authors' Contributions}

Chaoliang Tang was involved in the design and execution of experiments, data analysis, and manuscript writing. Zhetao Zhang and Fei Tang were involved in the design of the study, data analysis, and manuscript writing. Chengyun Hu, Feibiao Dai, Jiawu Wang, Lai Jiang, Di Wang, Jie Gao, Jun Huang, and Jianfeng Luo were all involved in the execution of experiments and data analysis. All authors provided final 
approval of the version to be submitted. Chengyun Hu, Feibiao Dai, and Jiawu Wang contributed equally to this work.

\section{Acknowledgments}

The authors are grateful for Mr. Kai Zhang's enthusiastic support and contribution to the design of graphics. This study was supported by grants from the National Natural Science Foundation of China (grant no. 81801175 to Chaoliang Tang), the China Postdoctoral Science Foundation (grant no. 2019M662179 to Chaoliang Tang), and the Anhui Province Postdoctoral Science Foundation (grant no. 2019B324 to Chaoliang Tang).

\section{References}

[1] S. A. Mohammed, B. Paramesha, Y. Kumar, U. Tariq, S. K. Arava, and S. K. Banerjee, "Allylmethylsulfide, a sulfur compound derived from garlic, attenuates isoproterenol-induced cardiac hypertrophy in rats," Oxidative Medicine and Cellular Longevity, vol. 2020, Article ID 7856318, 15 pages, 2020.

[2] J. Paavola, T. Alakoski, J. Ulvila et al., "Vezf1 regulates cardiac structure and contractile function," eBioMedicine, vol. 51, article 102608, 2020.

[3] J. Cao, D. B. Cowan, and D. Z. Wang, "tRNA-derived small RNAs and their potential roles in cardiac hypertrophy," Frontiers in Pharmacology, vol. 11, article 572941, 2020.

[4] M. Nakamura and J. Sadoshima, "Mechanisms of physiological and pathological cardiac hypertrophy," Nature Reviews. Cardiology, vol. 15, no. 7, pp. 387-407, 2018.

[5] C. Li, F. Liu, S. Liu et al., "Elevated myocardial SORBS2 and the underlying implications in left ventricular noncompaction cardiomyopathy," eBioMedicine, vol. 53, article 102695, 2020.

[6] N. Kastner, K. Zlabinger, A. Spannbauer et al., "New insights and current approaches in cardiac hypertrophy cell culture, tissue engineering models, and novel pathways involving non-coding RNA," Frontiers in Pharmacology, vol. 11, article 1314, 2020.

[7] S. Kumari, P. B. Katare, R. Elancheran et al., "Musa balbisiana fruit rich in polyphenols attenuates isoproterenol-induced cardiac hypertrophy in rats via inhibition of inflammation and oxidative stress," Oxidative Medicine and Cellular Longevity, vol. 2020, Article ID 7147498, 14 pages, 2020.

[8] C. B. Zheng, W. C. Gao, M. Xie et al., "Ang II promotes cardiac autophagy and hypertrophy via Orai 1/STIM1," Frontiers in Pharmacology, vol. 12, article 622774, 2021.

[9] G. Q. Zhang, S. Q. Wang, Y. Chen et al., "MicroRNAs regulating mitochondrial function in cardiac diseases," Frontiers in Pharmacology, vol. 12, article 663322, 2021.

[10] S. G. Rhee, H. A. Woo, and D. Kang, "The role of peroxiredoxins in the transduction of $\mathrm{H} 2 \mathrm{O} 2$ Signals," Antioxidants \& Redox Signaling, vol. 28, no. 7, pp. 537-557, 2018.

[11] S. Wang, L. Yu, G. Sun et al., "Danhong injection protects hemorrhagic brain by increasing peroxiredoxin 1 in aged rats," Frontiers in Pharmacology, vol. 11, p. 346, 2020.

[12] L. Jiang, Y. Gong, Y. Hu et al., "Peroxiredoxin-1 overexpression attenuates doxorubicin-induced cardiotoxicity by inhibiting oxidative stress and cardiomyocyte apoptosis," Oxidative Medicine and Cellular Longevity, vol. 2020, Article ID 2405135, 11 pages, 2020.
[13] S. Jaiswal, B. Joshi, J. Chen et al., "Membrane bound peroxiredoxin-1 serves as a biomarker forIn VivoDetection of sessile serrated adenomas," Antioxidants \& Redox Signaling, 2021.

[14] C. Tang, G. Yin, C. Huang et al., "Peroxiredoxin-1 ameliorates pressure overload-induced cardiac hypertrophy and fibrosis," Biomedicine \& Pharmacotherapy, vol. 129, article 110357, 2020.

[15] S. Matsushima, T. Ide, M. Yamato et al., "Overexpression of mitochondrial peroxiredoxin-3 prevents left ventricular remodeling and failure after myocardial infarction in mice," Circulation, vol. 113, no. 14, pp. 1779-1786, 2006.

[16] B. Knoops, J. Goemaere, V. Van der Eecken, and J. P. Declercq, "Peroxiredoxin 5: structure, mechanism, and function of the mammalian atypical 2-Cys peroxiredoxin," Antioxidants \& Redox Signaling, vol. 15, no. 3, pp. 817-829, 2011.

[17] Y. Ji, S. Chae, H. K. Lee et al., "Peroxiredoxin 5 controls vertebrate ciliogenesis by modulating mitochondrial reactive oxygen species," Antioxidants \& Redox Signaling, vol. 30, no. 14, pp. 1731-1745, 2019.

[18] Z. Wang, X. J. Zhang, Y. X. Ji et al., “The long noncoding RNA Chaer defines an epigenetic checkpoint in cardiac hypertrophy," Nature Medicine, vol. 22, no. 10, pp. 1131-1139, 2016.

[19] Y. Qiao, T. Hu, B. Yang et al., "Capsaicin Alleviates the Deteriorative Mitochondrial Function by Upregulating 14-3-3 $\eta$ in Anoxic or Anoxic/Reoxygenated Cardiomyocytes," Oxidative Medicine and Cellular Longevity, vol. 2020, Article ID 1750289, 16 pages, 2020.

[20] X. Chen, X. Cao, W. Xiao, B. Li, and Q. Xue, "PRDX5 as a novel binding partner in Nrf2-mediated NSCLC progression under oxidative stress," Aging, vol. 12, no. 1, pp. 122-137, 2020.

[21] M. H. Kim, H. J. Lee, S. R. Lee et al., "Peroxiredoxin 5 inhibits glutamate-induced neuronal cell death through the regulation of calcineurin-dependent mitochondrial dynamics in HT22 cells," Molecular and Cellular Biology, vol. 39, no. 20, 2019.

[22] F. Plaisant, A. Clippe, D. Vander Stricht, B. Knoops, and P. Gressens, "Recombinant peroxiredoxin 5 protects against excitotoxic brain lesions in newborn mice," Free Radical Biology \& Medicine, vol. 34, no. 7, pp. 862-872, 2003.

[23] M. H. Kim, S. J. Park, J. H. Kim et al., "Peroxiredoxin 5 regulates adipogenesis-attenuating oxidative stress in obese mouse models induced by a high-fat diet," Free Radical Biology \& Medicine, vol. 123, pp. 27-38, 2018.

[24] G. Wang, W. C. Zhong, Y. H. Bi et al., "The prognosis of peroxiredoxin family in breast cancer," Cancer Management and Research, vol. 11, pp. 9685-9699, 2019.

[25] R. D'Oria, R. Schipani, A. Leonardini et al., "The role of oxidative stress in cardiac disease: from physiological response to injury factor," Oxidative Medicine and Cellular Longevity, vol. 2020, Article ID 5732956, 29 pages, 2020.

[26] K. Hou, J. Shen, J. Yan et al., "Loss of TRIM21 alleviates cardiotoxicity by suppressing ferroptosis induced by the chemotherapeutic agent doxorubicin," eBioMedicine, vol. 69, article 103456, 2021.

[27] Q. Jin, Y. Jiang, L. Fu, Y. Zheng, Y. Ding, and Q. Liu, "Wenxin granule ameliorates hypoxia/reoxygenation-induced oxidative stress in mitochondria via the PKC-delta/NOX2/ROS pathway in H9c2 cells," Oxidative Medicine and Cellular Longevity, vol. 2020, Article ID 3245483, 16 pages, 2020.

[28] X. H. Xie, J. B. Liao, F. Fang et al., "Jian-Gan-Xiao-Zhi decoction ameliorates high-fat high-carbohydrate diet-induced 
non-alcoholic fatty liver disease and insulin resistance by regulating the AMPK/JNK pathway," Traditional Medicine Research, vol. 6, no. 1, p. 4, 2021.

[29] A. J. P. O. de Almeida, M. S. de Almeida Rezende, S. H. Dantas et al., "Unveiling the role of inflammation and oxidative stress on age-related cardiovascular diseases," Oxidative Medicine and Cellular Longevity, vol. 2020, Article ID 1954398, 20 pages, 2020.

[30] A. W. Rookyard, J. Paulech, S. Thyssen et al., "A global profile of reversible and irreversible cysteine redox post-translational modifications during myocardial ischemia/reperfusion injury and antioxidant intervention," Antioxidants \& Redox Signaling, vol. 34, no. 1, pp. 11-31, 2021.

[31] Z. Cai, Z. Wang, R. Yuan et al., "Redox-sensitive enzyme SENP3 mediates vascular remodeling via de-SUMOylation of $\beta$-catenin and regulation of its stability," eBioMedicine, vol. 67, article 103386, 2021.

[32] S. Peng, X. F. Lu, Y. D. Qi et al., "LCZ696 Ameliorates Oxidative Stress and Pressure Overload-Induced Pathological Cardiac Remodeling by Regulating the Sirt3/MnSOD Pathway," Oxidative Medicine and Cellular Longevity, vol. 2020, Article ID 9815039, 15 pages, 2020.

[33] M. Zhang, Y. Xu, J. Chen et al., "Beta3-Adrenergic receptor activation alleviates cardiac dysfunction in cardiac hypertrophy by regulating oxidative stress," Oxidative Medicine and Cellular Longevity, vol. 2021, Article ID 3417242, 13 pages, 2021.

[34] X. Yan, Q. Y. Zhang, Y. L. Zhang, X. Han, S. B. Guo, and H. H. $\mathrm{Li}$, "Gallic acid attenuates angiotensin II-induced hypertension and vascular dysfunction by inhibiting the degradation of endothelial nitric oxide synthase," Frontiers in Pharmacology, vol. 11, p. 1121, 2020.

[35] L. Gao, A. Feng, C. Li, S. Schmull, and H. Sun, "Decellularized aortic scaffold alleviates $\mathrm{H}_{2} \mathrm{O}_{2}$-induced inflammation and apoptosis in CD34+ progenitor cells while driving neovasculogenesis," BioMed Research International, vol. 2020, Article ID 6782072, 10 pages, 2020.

[36] W. Y. Abdelzaher, S. M. Ahmed, N. N. Welson, K. F. Alsharif, G. E. Batiha, and D. A. A. Labib, "Dapsone ameliorates isoproterenol-induced myocardial infarction via Nrf2/ HO-1; TLR4/ TNF- $\alpha$ signaling pathways and the suppression of oxidative stress, inflammation, and apoptosis in rats," Frontiers in Pharmacology, vol. 12, article 669679, 2021.

[37] Y. Liang, Q. Q. Niu, and Y. H. Zhao, "Pharmacological research progress of ursolic acid for the treatment of liver diseases," Traditional Medicine Research, vol. 6, no. 4, p. 38, 2021.

[38] Y. Cheng, A. Shen, X. Wu et al., "Qingda granule attenuates angiotensin II-induced cardiac hypertrophy and apoptosis and modulates the PI3K/AKT pathway," Biomedicine \& Pharmacotherapy, vol. 133, article 111022, 2021.

[39] M. Jin, H. Yu, X. Jin, L. Yan, J. Wang, and Z. Wang, “Dracocephalum moldavica $\mathrm{L}$. extracts protect $\mathrm{H} 9 \mathrm{c} 2$ cardiomyocytes against $\mathrm{H}_{2} \mathrm{O}_{2}$-induced apoptosis and oxidative stress," BioMed Research International, vol. 2020, Article ID 8379358, 12 pages, 2020.

[40] M. Yuan, M. Gong, Z. Zhang et al., "Hyperglycemia induces endoplasmic reticulum stress in atrial cardiomyocytes, and mitofusin-2 downregulation prevents mitochondrial dysfunction and subsequent cell death," Oxidative Medicine and Cellular Longevity, vol. 2020, Article ID 6569728, 14 pages, 2020.

[41] Y. T. Wu, L. P. Xie, Y. Hua et al., "Tanshinone I inhibits oxidative stress-induced cardiomyocyte injury by modulating $\mathrm{Nrf} 2$ signaling," Frontiers in Pharmacology, vol. 12, article 644116, 2021.

[42] G. P. Caviglia, "Edaravone and MAPK pathway: the key role of gut permeability," Minerva Medica, vol. 111, no. 2, p. 181, 2020.

[43] Q. Ling, F. Li, X. Zhang et al., "MAP4K1 functions as a tumor promotor and drug mediator for AML via modulation of DNA damage/repair system and MAPK pathway," eBioMedicine, vol. 69, article 103441, 2021.

[44] H. N. Siti, J. Jalil, A. Y. Asmadi, and Y. Kamisah, "Parkia speciosa Hassk. Empty pod extract alleviates angiotensin IIinduced cardiomyocyte hypertrophy in $\mathrm{H} 9 \mathrm{c} 2$ cells by modulating the Ang II/ROS/NO Axis and MAPK pathway," Frontiers in Pharmacology, vol. 12, article 741623, 2021.

[45] T. Zhao, H. J. Kee, L. Bai, M. K. Kim, S. J. Kee, and M. H. Jeong, "Selective HDAC8 inhibition attenuates isoproterenolinduced cardiac hypertrophy and fibrosis via p 38 MAPK pathway," Frontiers in Pharmacology, vol. 12, article 677757, 2021.

[46] S. Tamura, T. Marunouchi, and K. Tanonaka, "Heat-shock protein 90 modulates cardiac ventricular hypertrophy via activation of MAPK pathway," Journal of Molecular and Cellular Cardiology, vol. 127, pp. 134-142, 2019. 